\title{
An ultrasound transient elastography system with coded excitation
}

\author{
Xianfen Diao, Jing Zhu, Xiaonian He, Xin Chen, Xinyu Zhang, Siping Chen and Weixiang Liu*
}

*Correspondence:
wxliu@szu.edu.cn
National-Regional Key
Technology Engineering
Laboratory for Medical
Ultrasound, Guangdong Key
Laboratory for Biomedical
Measurements
and Ultrasound Imaging,
Department of Biomedical
Engineering, School
of Medicine, Shenzhen
University, Shenzhen 518060,
China

*Correspondence:

wxliu@szu.edu.cn

Technology Engineering

Laboratory for Medical

Ultrasound, Guangdong Key

Laboratory for Biomedica

Engineering, School

University, Shenzhen 518060

China

\begin{abstract}
Background: Ultrasound transient elastography technology has found its place in elastography because it is safe and easy to operate. However, it's application in deep tissue is limited. The aim of this study is to design an ultrasound transient elastography system with coded excitation to obtain greater detection depth.

Methods: The ultrasound transient elastography system requires tissue vibration to be strictly synchronous with ultrasound detection. Therefore, an ultrasound transient elastography system with coded excitation was designed. A central component of this transient elastography system was an arbitrary waveform generator with multi-channel signals output function. This arbitrary waveform generator was used to produce the tissue vibration signal, the ultrasound detection signal and the synchronous triggering signal of the radio frequency data acquisition system. The arbitrary waveform generator can produce different forms of vibration waveform to induce different shear wave propagation in the tissue. Moreover, it can achieve either traditional pulse-echo detection or a phase-modulated or a frequency-modulated coded excitation. A 7-chip Barker code and traditional pulse-echo detection were programmed on the designed ultrasound transient elastography system to detect the shear wave in the phantom excited by the mechanical vibrator. Then an elasticity QA phantom and sixteen in vitro rat livers were used for performance evaluation of the two detection pulses.
\end{abstract}

Results: The elasticity QA phantom's results show that our system is effective, and the rat liver results show the detection depth can be increased more than $1 \mathrm{~cm}$. In addition, the SNR (signal-to-noise ratio) is increased by $15 \mathrm{~dB}$ using the 7-chip Barker coded excitation.

Conclusions: Applying 7-chip Barker coded excitation technique to the ultrasound transient elastography can increase the detection depth and SNR. Using coded excitation technology to assess the human liver, especially in obese patients, may be a good choice.

Keywords: Ultrasound transient elastography, Coded excitation, Detection depth

\section{Background}

Tissue elasticity and viscosity are closely related to pathological changes. Therefore, quantitative measurement of tissue viscoelasticity has important medical applications. The viscoelasticity measurement technology includes three steps. First, an internal or external dynamic, static, or quasi-static force is applied to the tissue to produce microdeformation or shear waves in the tissue. Second, an ultrasound imaging technique, 
magnetic resonance imaging (MRI), or optical imaging is used to detect the elastic deformation, the shear wave amplitude, speed, or phase et al. Third, a mathematical model correlating the elasticity, viscosity, density and shear wave information is used to deduce the elasticity and viscosity modulus.

Several ultrasound elasticity techniques with different tissue vibrations have been reported in the past 20 years, including intravascular ultrasound elastography (IVUSE) [1], quasi-static ultrasound elastography [2-4], acoustic radiation force impulse imaging (ARFI) [5, 6], ultrasound vibro-acoustic imaging (USVA) [7, 8], shear wave dispersion ultrasound vibrometry (SDUV) [9], supersonic shear imaging (SSI) [10], external vibration transient elastography [11-13] and so on. Ultrasound-based elastography has the advantages of real-time, noninvasive, low-cost, et al. However, ultrasound-based elastographies have a common defect, in that the detection depth is limited because the ultrasound waves attenuate, and low amplitude shear waves attenuate quickly in the tissue.

One of the clinical applications of ultrasound elasticity technique is to quantitative evaluation of liver, for example, hepatic steatosis, liver fibrosis and cirrhosis. About $50-80 \%$ obese patients [14] and $86-96 \%$ severely overweight people [15] are liver steatosis. However, ultrasound-based elastographies cannot provide robust shear wave motion detection in the obese patients. The shear wave elastography using the plane wave imaging also suffers from poor penetration [16]. Recently, Echosens develop a probe dedicated to obese patients and controlled attenuation parameter is developed to assess liver steatosis, too [17]. Lai reports that there are about one-third of obese patients have unreliable liver stiffness value measured by vibration-controlled transient elastography by Fibroscan [18]. Thus, it is necessary to develop the ultrasound-based elastography with bigger penetration.

One way to increase the detection depth is to apply coded excitation. There have been several research studies on coded excitation in an elastography technique. Peng and Liu applied coded excitation in the quasi-static elastography and shows that the SNR estimation displacement can be improved, and that the strain noise can be reduced. And they apply chirp-coded pulse detection into a real-time ultrasound elastography system, experimental results also show that the SNR of strain image is increased [19, 20]. Song et al. using a 13-bit Barker code, a short chirp and a long chirp for shear wave detection, results show that the penetration depth can be increased 2,3 , and $4 \mathrm{~cm}$ respectively without decreasing the frame rate, field-of-view of plane wave imaging [16]. Although their studies prove that the coded excitation is an important tool to enhance performance of medical ultrasound elastography, little attention has been paid to the effect of using coded excitation on the external vibration transient elastography.

In this study we tested the feasibility of using coded excitation on the external vibration transient elastography. This paper investigates the application of coded excitation on external vibration transient elastography. An external vibration transient elastography system with coded excitation was designed. An elasticity QA phantom (Model 049CIRS Inc., Norfolk, VA) and in vitro rat livers were used to test the performance of our system. The elasticity QA phantom's results show that our system is effective, and the rat livers' results show that penetration depth and SNR are noticeably improved when using Barker coded excitation on the transient elastography system. 


\section{Viscoelasticity mathematical model}

For a homogeneous medium, the viscoelasticity mathematical model is as follow [21]:

$$
c_{s}=\sqrt{\frac{2\left(\mu_{1}^{2}+\omega^{2} \mu_{2}^{2}\right)}{\rho\left(\mu_{1}+\sqrt{\mu_{1}^{2}+\omega^{2} \mu_{2}^{2}}\right)}}
$$

where $c_{s}$ is shear wave propagation speed; $\mu_{1}$ and $\mu_{2}$ are shear elasticity and shear viscosity of the medium, respectively; $\rho$ is the mass density of the medium (most soft tissues $\rho=1 \mathrm{~kg} / \mathrm{m}^{3}$ [22]); $\omega$ is the frequency of shear wave. However, since not all of the tissues are homogeneous, other models are used to measure elasticity and viscosity, and will result in different elasticity and viscosity values. Suppose shear viscosity is zero, that is, $\mu_{2}=0$, we get the linear elastic model as follows:

$$
c_{s}=\sqrt{\frac{\mu_{1}}{\rho}}
$$

where the shear wave propagation speed $c_{s}$ is called group velocity. If the mass density $\rho$ of the tissue is the same, there is one-to-one relation between the group velocity $c_{s}$ and the shear elasticity $\mu_{1}$ of the medium. Therefore, in our paper we used shear wave speed on behalf of elasticity.

\section{Experiment setup}

The system setup with arbitrary vibration waveform and coded excitation is shown in Fig. 1. The external vibration transient elastography system consists of two parts: tissue vibration and pulse-echo ultrasound detection. The tissue vibration section is used to generate shear waves in medium, and the pulse-echo ultrasound detection section is responsible for detecting weak vibration information. Their details are described as follows:

\section{Tissue vibration}

As shown in Fig. 1 the tissue vibration part consists of an arbitrary waveform generator (LeCroy Arbstudio 1104, Leroy Corp., USA), a power amplifier (Type 2718, B\&K, Denmark), a small mechanical vibrator (Mini-shaker Type 4810, B\&K, Denmark), a transducer ( $2 \mathrm{MHz}$ ), and an elongated bar. The arbitrary waveform generator can generate a specified vibration signal, and then employ a power amplifier to drive the mechanical vibrator to excite tissue through the elongated bar. The small mechanical vibrator has a frequency range from direct current to $18 \mathrm{kHz}$, which is enough for elasticity study. The arbitrary waveform generator can produce tissue vibration waveform as required. For example, one period sine wave can be used at different frequencies to excite tissue, similar to suddenly beating the tissue; $N$ cycles of sine pulses or a continuous vibration waveform at a frequency can also be used. Different forms of vibration waveform induce different shear wave propagation in the tissue, which is useful for measuring the viscoelasticity of tissue. 


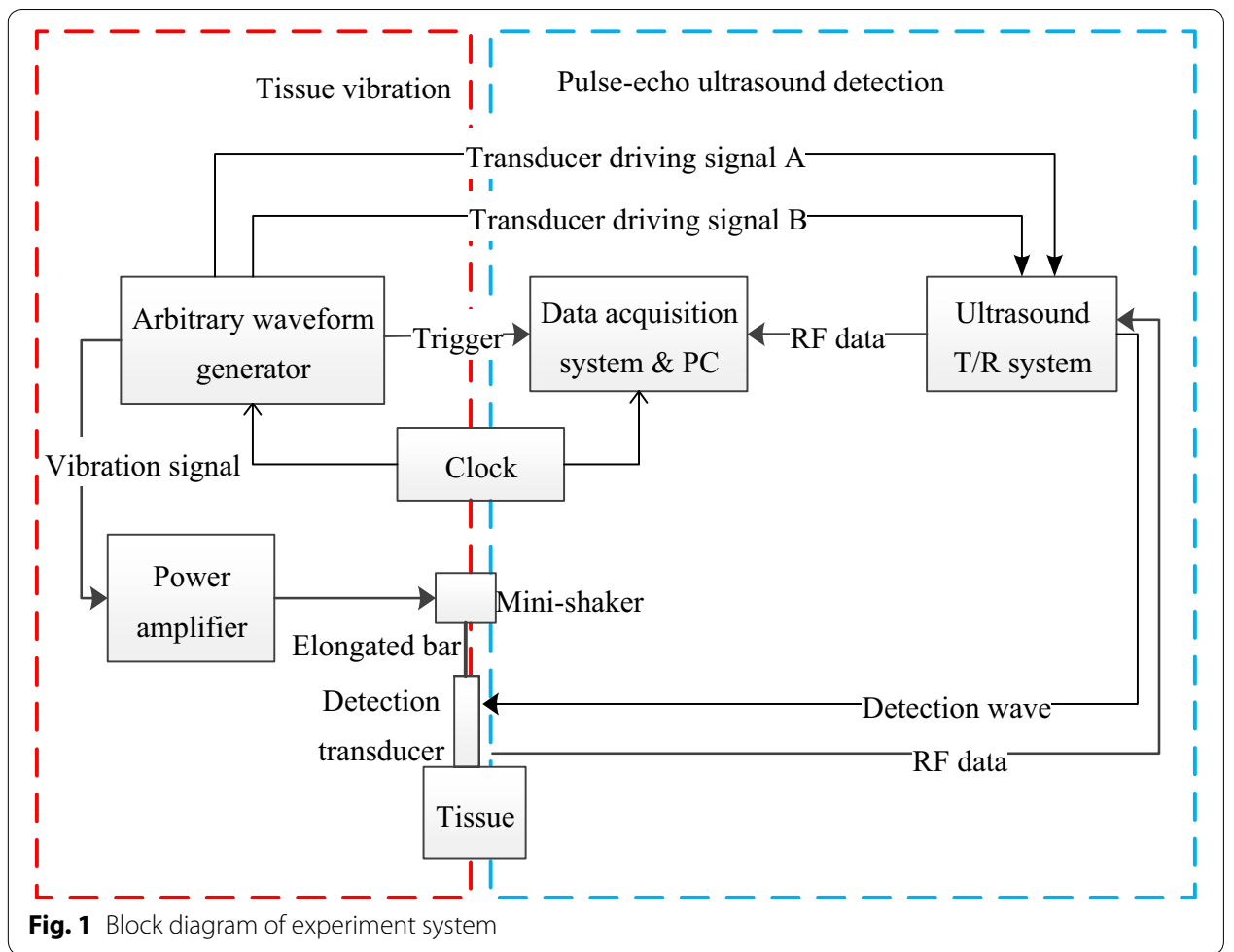

\section{Pulse-echo ultrasound detection}

Pulse-echo ultrasound detection shown in Fig. 1 is composed of an arbitrary waveform generator, an ultrasound T/R system, a transducer, a data acquisition system (PCI-9846, ADLINK), and a PC. The arbitrary waveform generator generates two signals to drive the transducer. Likewise, the transducer driving signals produced by the arbitrary waveform generator are very flexible. Consequently, it can achieve either traditional pulseecho detection or coded excitation. The radio frequency (RF) signals are received by the transducer and captured by the data acquisition system. The sampled RF data are processed offline in the Matlab (The MathWorks, Inc., Natick, MA, USA) environment.

In a conventional Doppler ultrasound system, the transmitting pulse contains several cycles of sine wave whose frequency is identical to the central frequency of the transducer. However, coded excitation in a Doppler ultrasound system must transmit a phase-modulated waveform or a frequency-modulated waveform. Here, the arbitrary waveform generator is chosen to produce a phase-modulated waveform. For the preliminary experiment, a 7-chip Barker code was used for the coded excitation in our study and every chip of the Barker code consisted of four sine waves, abbreviated as 7c4w. The traditional pulse-echo detection emitted four sine cycles with a frequency of $2 \mathrm{MHz}$. The pulse repetition frequency was $7.4 \mathrm{kHz}$.

Here, the scatter's vibration with time was described by the slow time signal. The slow time signal's extracted algorithm was written by referring to the Doppler principle [23]. Shear wave speed can be calculated by the different peak time [21, 24], used in the FibroScan system. The data processing procedure is shown in Fig. 2. When coded excitation mode is used, a matched filter was chosen for decoding, that is, the correlation 


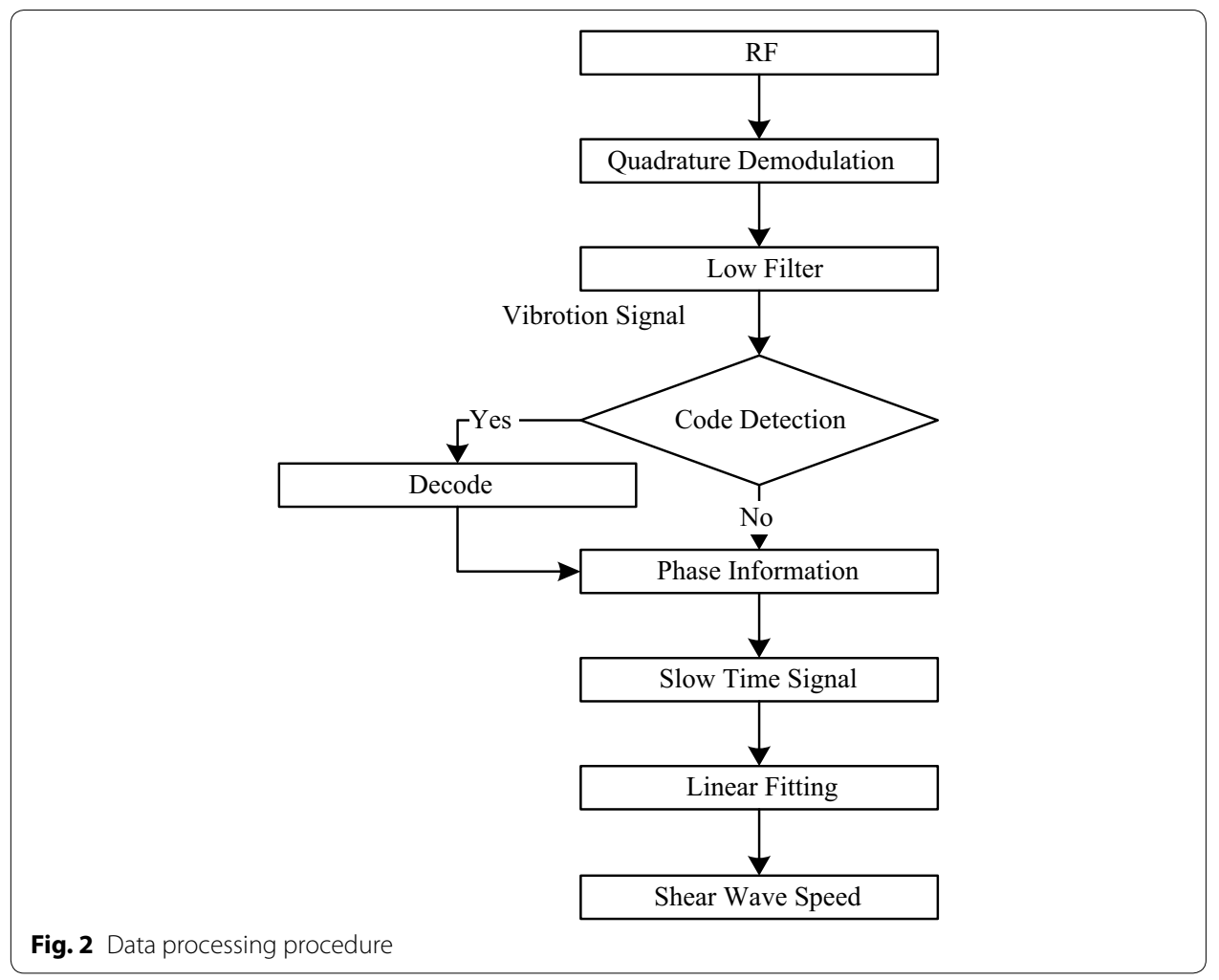

calculations of the coded sequence and ultrasound echoes through multiplication and summation.

An ultrasound elastography system requires tissue vibration strictly synchronous with pulse-echo ultrasound detection. As shown in Fig. 1, the tissue vibration portion and the pulse-echo ultrasound detection portion work under the same clock source, which was $8 \mathrm{MHz}$ for the whole system. It provided the clock signal to the arbitrary waveform generator and the data acquisition system. Tissue vibration signals, transducer driving signals, and the trigger signal of the data acquisition system were all produced by the arbitrary waveform generator. Therefore, the synchronization of the system could be achieved easily by ensuring that these three signals had a public cycle.

\section{Evaluation of system performance}

\section{Elasticity QA phantom study}

The developed transient elastography system was used first to measure the elasticity QA phantom, and the results were used to evaluate the system's accuracy. The tissue vibration signal was a single sine wave, and the pulse width was $8.6 \mathrm{~ms}$; the pulse repetition frequency was $7.4 \mathrm{kHz}$ (results shown in Fig. 3). Figure 3b depicts the overall effect map acquired by drawing the slow time signals of different depths in one picture. This result revealed the vibration propagation process from shallow to deep. Figure 3a depicts the correspondence between the peak time and depth. The deep range was between 2 and $7 \mathrm{~cm}$ under the surface. The line was the result obtained by curve fitting, whose slope was 2.7 , this meant that the shear wave speed was $2.7 \mathrm{~m} / \mathrm{s}$. Under the same conditions we detected ten points, and the shear wave speed result was $2.9 \pm 0.18 \mathrm{~m} / \mathrm{s}$. The normal 


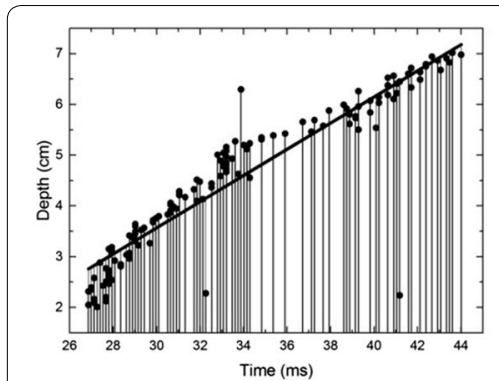

$\mathbf{a}$

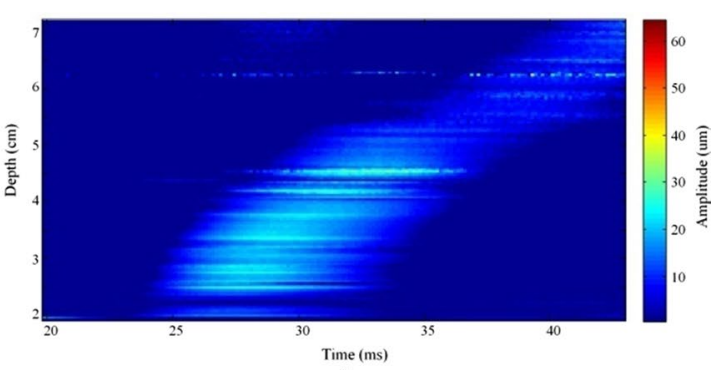

b

Fig. 3 a The correspondence between the peak time and depth; $\mathbf{b}$ The overall trend of the shear wave propagation in the elasticity QA phantom

value of the elasticity QA phantom is $2.7-3.1 \mathrm{~m} / \mathrm{s}$. The elasticity value measured by our system was within the rational range, which indicated that our system was effective.

\section{Rat liver study}

The rat livers were also used to evaluate system performance. Sixteen rats, raised by Guangdong Medical Laboratory Animal Center under identically stable conditions, were used in our experiments. The rat liver was embedded in a phantom, and the distance between the liver and the surface was about $2 \mathrm{~cm}$. The phantom was made from gelatin and water with a 1:9 ratio. All procedures in these studies were approved by Animal Care Committee of Shenzhen University and Guangdong Medical Laboratory Animal Center.

Figure 4 gives the slow time signals obtained by one of the rat liver at $5.5-6 \mathrm{~cm}$ depth using $7 \mathrm{c} 4 \mathrm{w}$ coded excitation and traditional pulse-echo detection. A single sine impulse with a pulse width of $8.6 \mathrm{~ms}$ was used as the vibration signal. Obviously, the slow time signals obtained by using $7 \mathrm{c} 4 \mathrm{w}$ coded excitation were more regular. These results show that the $7 \mathrm{c} 4 \mathrm{w}$ coded excitation had better stability and anti-interference ability than traditional detection. In fact, coded excitation can increase the signal-to-noise ratio because the white noise in the echoes is reduced through the correlation calculations in the decoding process. Additionally, due to the equipment delay, the main vibration period was about $18 \mathrm{~ms}$, which was very different from the vibration pulse width. The second peak was used to calculate the shear wave speed.

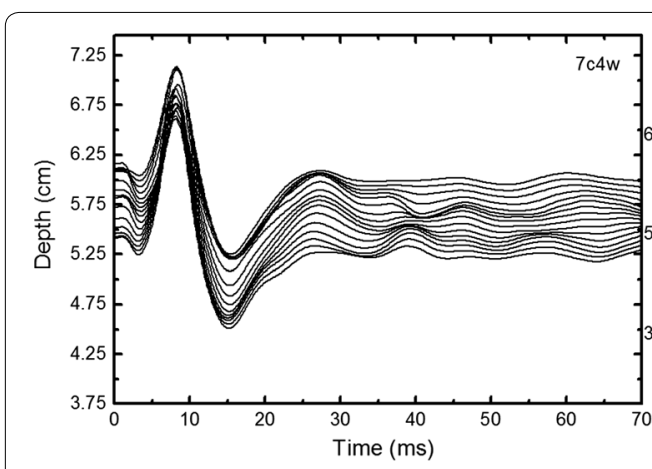

a

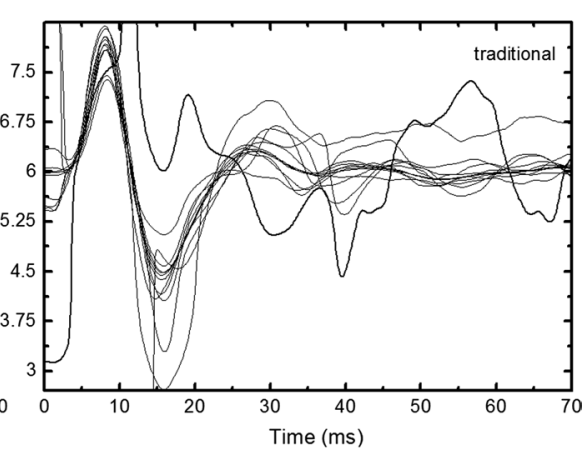

b

Fig. 4 Slow time signals at $5.5-6 \mathrm{~cm}$ depth obtained by using different detection methods: $\mathbf{a}$ is using the 7-chip Barker coded detection, and $\mathbf{b}$ is using traditional pulse-echo detection 
For coded excitation, the corresponding matched filters were used in the offline signals process algorithm. An obvious difference existed relating to the signal amplitude and the initial phase between the results obtained by using $7 \mathrm{c} 4 \mathrm{w}$ and traditional detection. The difference has no influence on the measurement of shear wave speed, regardless of which difference (phase or peak time) is used to deduce the shear wave speed, the subtraction operation will remove the bias.

Figure 5 shows the entire trend of slow time signals in one of the rat livers. It was clear that the shear wave propagation was below $5 \mu \mathrm{m}$ over $4.5 \mathrm{~cm}$ in Fig. $5 \mathrm{~b}$, while the shear wave propagation process detected by using the $7 \mathrm{c} 4 \mathrm{w}$ coded excitation was still about $20 \mu \mathrm{m}$ in the depth between 4.5 and $5.5 \mathrm{~cm}$ in Fig. 4c. The vibration amplitudes detected by 7c4w coded excitation were much bigger than those detected by traditional pulse-echo detection because there were multiplication and summation computation in the decoding algorithm. In addition, as shown by the results in Fig. $5 c$, the attenuation of the slow time signal amplitude was not obvious when depth was increased, which demonstrated the superiority of the coded excitation in measuring the weak signals.

We detected three points from each rat liver, and then averaged those points after outlier removal. The shear wave speeds of the rat livers in different detection modes were shown in Table 1. A T test showed that the shear wave speed was not significantly different $(p>0.05)$ between traditional detection and 7c4w detection. Results of sixteen rats show that the 7c4w coded excitation can improve the penetration depth by about $1 \mathrm{~cm}$ when the vibration amplitude is only several microns and the SNR is increased about $15 \mathrm{~dB}$.

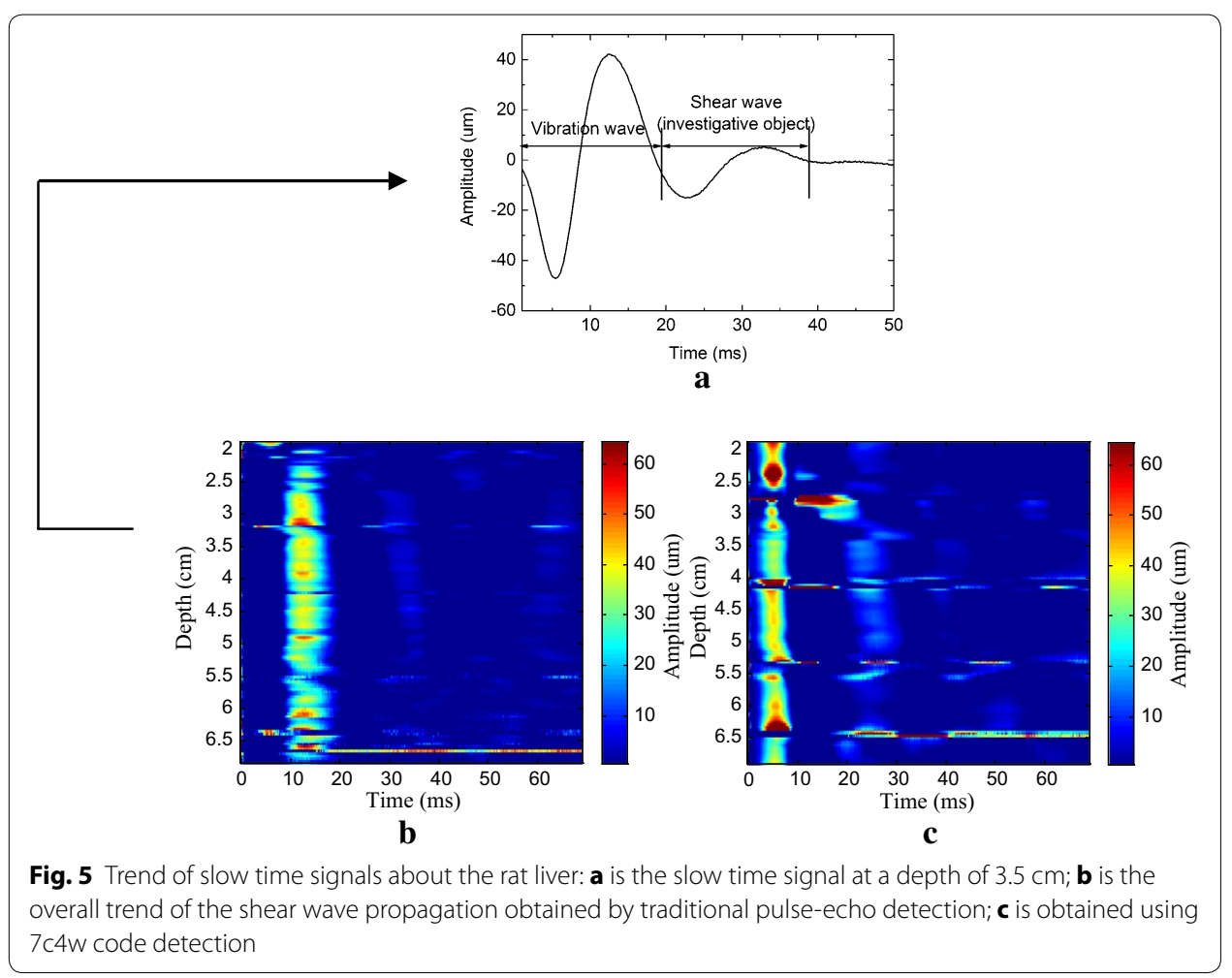


Table 1 Shear wave speed in the rat livers obtained by different detection modes

\begin{tabular}{lc}
\hline Detection mode & Shear wave speed $(\mathbf{m} / \mathbf{s})$ \\
\hline Traditional & $1.89 \pm 0.58$ \\
$7 \mathrm{c} 4 \mathrm{~W}$ & $2.02 \pm 0.98$ \\
\hline
\end{tabular}

\section{Discussion}

In our experiments, the tissue vibration signal is a single sine wave, therefore the slow time signal representing tissue vibration is shock attenuation curve. The first peak of the slow time signal is obviously stronger than the second, as shown in Fig. 5a. But the second peak of the slow time signal is used to deduce the shear wave speed. This is because the slow time signal besides the first peak includes the tissue vibration and transducer moving information. If using the first peak of the slow time signal to calculate the shear wave speed, a new algorithm needs to be designed to remove the transducer moving information from the extracted slow time signal. The next work is to design such an algorithm.

This study investigates the feasibility of using coded excitation applied to the external vibration ultrasound transient elastography. The performance of coded excitation and traditional pulse-echo detection are compared using the penetration depth and signal-to-noise ratio (SNR) which are the two main technical specifications of ultrasound elastography. Next, the in vivo study will be done to compare the performance of our ultrasound transient elastography system in quantifying the liver stiffness for the obese. Besides, different coded excitation waveforms or encoding filters need to be investigated to further improve the performance of the coded excitation.

\section{Conclusions}

An ultrasound transient elastography system with coded excitation is developed. The rigorous synchronization of the transient elastography system and coded excitation is executed by using an arbitrary waveform generator. The flexibility of the arbitrary waveform generator ensures that traditional pulse-echo detection, coded excitation, and different tissue vibration waveforms can be realized. Experiment results suggest that higher penetration depth and SNR can be achieved using the 7-chip Barker coded excitation for the ultrasound transient elastography system.

\section{Abbreviations}

SNR: signal-to-noise ratio; MRI: magnetic resonance imaging; IVUSE: intravascular ultrasound elastography; ARFI: acoustic radiation force impulse imaging; USVA: ultrasound vibro-acoustic imaging; SDUV: shear wave dispersion ultrasound vibrometry; SSI: supersonic shear imaging; RF: radio frequency; 7c4w: 7-chip Barker code and every chip of the Barker code consisted of four sine waves.

\section{Authors' contributions}

XD carried out the experiment system design, the elasticity QA phantom studies. JZ and XH carried out the system putup and rat liver study. XC carried out the system debugging. XZ carried out the rat liver phantom madeup. SC participated the signal processing work. WL suggested the whole plan of this study and participated in the design of the study. All authors read and approved the final manuscript. 


\section{Competing interests}

The authors declare that they have no competing interests.

Availability of data and materials

If anyone wants the original RF data, he/she can send an e-mail to laodiao@szu.edu.cn ask for the data.

\section{Consent for publication}

All authors consent to publish this paper.

\section{Ethics approval and consent to participate}

All procedures in these studies were approved by Animal Care Committee of Shenzhen University, Guangdong, China and Guangdong Medical Laboratory Animal Center, Guangdong, China.

\section{Funding}

This research was supported in part by National Natural Science Foundations of China (Grants 61201041, 61427806), in part by the Science and Technology Planning Project of Shenzhen City (Grant JCYJ20150525092941053), in part by the Medical Scientific Research Foundation of Guangdong Province (Grant A2017280).

\section{Publisher's Note}

Springer Nature remains neutral with regard to jurisdictional claims in published maps and institutional affiliations.

Received: 31 March 2017 Accepted: 20 June 2017

Published online: 28 June 2017

\section{References}

1. Liu K, Zhu XJ, Shao JH, Bai J, Zhang PF. Research on comparison of imaging parameters in intravascular ultrasound elastography. Space Med Med Eng. 2010;23:147-52.

2. Treece GM, Lindop JE, Gee AH, Prager RW. Freehand ultrasound elastography with a 3-D probe. Ultrasound Med Biol. 2008;34:463-74.

3. Ginat DT, Destounis SV, Barr RG, Castaneda B, Strang JG, Rubens DJ. US elastography of breast and prostate lesions. Radiographics a review publication of the radiological society of North America Inc. 2009; 29:2007-16.

4. Zheng YP, Zhou YJ. An implementation of ultrasonic tissue elastography in a conventional ultrasound scanner. China Med Dev. 2011;26(1):1-3.

5. Nightingale K, Soo MS, Nightingale R, Trahey G. Acoustic radiation force impulse imaging: in vivo demonstration of clinical feasibility. Ultrasound Med Biol. 2002;28:227.

6. Tierney ÁP, Callanan A, McGloughlin TM. In vivo feasibility case study for evaluating abdominal aortic aneurysm tissue properties and rupture potential using acoustic radiation force impulse imaging. J Mech Behav Biomed Mater. 2011;4:507.

7. Fatemi M, Greenleaf JF. Ultrasound-stimulated vibro-acoustic spectrography. Science. 1998;280:82.

8. Zheng $\mathrm{Y}$, Chen $\mathrm{S}$, Tan W, Kinnick R. Detection of tissue harmonic motion induced by ultrasonic radiation force using pulse-echo ultrasound and kalman filter. IEEE Trans Ultrason Ferroelectr Freq Control. 2007;54:290-300.

9. Mitri FG, Urban MW, Fatemi M, Greenleaf JF. Shear wave dispersion ultrasonic vibrometry for measuring prostate shear stiffness and viscosity: an in vitro pilot study. IEEE Trans Bio-med Eng. 2011;58:235.

10. Bercoff J, Tanter M, Fink M. Supersonic shear imaging: a new technique for soft tissue elasticity mapping. IEEE Trans Ultrason Ferroelectr Freq Control. 2004;51:396-409.

11. Lerner RM, Parker KJ, Holen J, Gramiak R, Waag RC. Sono-elasticity: medical elasticity images derived from ultrasound signals in mechanically vibrated targets. US: Springer; 1988.

12. Li Y. The study of sonoelasticity imaging of soft tissue using ultrasound Doppler technique. Ying Yong LI Xue Xue Bao/Chin J Appl Mech. 1999; 16.

13. Yamakoshi Y, Otaki H, Shinozuka N, Masuda H. Internal tissue displacement measurement based on ultrasonic wave Doppler signal digital detection and its application to fetal movement monitoring. Ultrasonics. 1996;34:769-75.

14. Farrell GC, Larter CZ. Nonalcoholic fatty liver disease: from steatosis to cirrhosis. Hepatology. 2006;43:S99.

15. Gholam PM, Flancbaum L, Machan JT, Charney DA, Kotler DP. Nonalcoholic fatty liver disease in severely obese subjects. Am J Gastroenterol. 2007;102:399-408.

16. Song P, Urban MW, Manduca A, Greenleaf JF, Chen S. 2083648 robust and ultrafast detection of shear wave motion using coded excitation. Ultrasound Med Biol. 2015;41:S97-8.

17. Sasso M, Audière S, Kemgang A, Gaouar F, Corpechot C, Chazouillères $O$, et al. Liver steatosis assessed by controlled attenuation parameter (CAP) measured with the XL probe of the FibroScan: a pilot study assessing diagnostic accuracy. Ultrasound Med Biol. 2016;42:92.

18. Lai M. Is liver stiffness measurement to stage fibrosis in patients with nonalcoholic fatty liver disease ready for clinical use? Hepatology. 2015;62:997-8.

19. Peng H, Liu DC. Chirp-coded pulse excitation for ultrasound elasticity imaging. In: International Conference on bioinformatics and biomedical engineering; 2010. p. 1-4.

20. Peng H, Liu DC. Enhanced ultrasound strain imaging using chirp-coded pulse excitation. Biomed Signal Process Control. 2011;8:130-41.

21. Catheline S, Thomas JL, Wu F, Fink MA. Diffraction field of a low frequency vibrator in soft tissues using transient elastography. IEEE Trans Ultrason Ferroelectr Freq Control. 1999;46:1013. 
22. Partin A, Hah Z, Barry CT, Rubens DJ, Parker KJ. Elasticity estimates from images of crawling waves generated by miniature surface sources. Ultrasound Med Biol. 2014;40:685-94.

23. Zheng Y, Chen S, Zhang X, Greenleaf JF. Detection of shear wave propagation in an artery using pulse echo ultrasound and Kalman filtering. In: ultrasonics symposium, vol. 1252; 2004. p. 1251-53.

24. Catheline S, Fink M. A solution to diffraction biases in sonoelasticity: the acoustic impulse technique. J Acoust Soc Am. 1999;105:2941.

Submit your next manuscript to BioMed Central and we will help you at every step:

- We accept pre-submission inquiries

- Our selector tool helps you to find the most relevant journal

- We provide round the clock customer support

- Convenient online submission

- Thorough peer review

- Inclusion in PubMed and all major indexing services

- Maximum visibility for your research

Submit your manuscript at www.biomedcentral.com/submit 